\title{
Research Based on Cohesive Zone Model for Interface Delamination of Waste Plastic Chip
}

\author{
Xueping Liu, a , Zufu Pang ${ }^{1,2, b}$, Dong Xiang ${ }^{2, c}$, Yongkai Zhang ${ }^{2, d}$ \\ ${ }^{1}$ Graduate School at Shenzhen, Tsinghua University, Shenzhen 518055 \\ 2 Department of Mechanical Engineering, Tsinghua University, Beijing 100084 \\ ${ }^{a}$ liuxp@sz.tsinghua.edu.cn, b pzf08@foxmail.com,

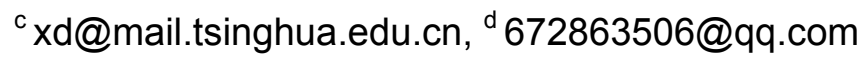

Keywords: PCB disassembly; plastic chip delamination; Cohesive Zone Model; simulation analysis

\begin{abstract}
The interface delamination of waste plastic chip will seriously affect the value of their recycling reuse; therefore, it has been a hot issue in recycling researches of Waste Electrical and Electronic Equipment (WEEE). Based on the exponential cohesion theory, this paper has researched chip delamination and established the Cohesive Zone Model (CZM) of delamination crack for chip interface, as well as proposed a method which combined the experiment of crack surface observation and the simulation of fracture mechanics. By employing this method, the CZM parameters of waste plastic chip interface have been obtained. Based on the CZM, the simulation of waste plastic chip interface delamination has been made, and its results showed that: temperature and humidity factors have a significant impact on the chip delamination. At last, the disassembly experiments of Waste Printed Circuit Boards (WPCB) have been made under different disassembly technology, and the delamination rate of resulting chips has been calculated. Then this paper has analyzed the influence rule of different disassembly conditions on the chip delamination and verified the correctness of chip delamination simulation results, as well as proposed an optimized disassembly technology of WPCB for reducing plastic chip's delamination.
\end{abstract}

\section{Introduction}

With the rapid development of world economy, a large number of Waste Electrical and Electronic Equipment (WEEE) appeared each year, and caused tremendous pressure to the environment, then the recycling of WEEE has become a major issues causing social attention. Countries all around the world have issued corresponding bill about WEEE processing ${ }^{[1]}$. For example, the WEEE Directive issued by European Union in 2003 cleared rules for ten classes of recovery and regeneration utilization of WEEE. Printed Circuit Board (PCB) is the important material for classification processing after dismantling the WEEE, "the National Hazardous Waste List" of China clearly stipulates that PCB components are T-class hazardous wastes. Therefore, studying the recycling method is of great significance. Component reuse is an effective method to achieve the high value recycling of Waste Printed Circuit Boards (WPCB), and is the current hot research spot.

Study shows that the design life of components is 500000 hours, while the average service life of WEEE in developed country is only 20000 hours. According to the Bathtub Curve theory of components invalidation, the component is in the optimal period of quality, and has high reuse value ${ }^{[2]}$. Guo Xiuying et al. ${ }^{[3,4]}$ researched the reusability of waste plastic chip through experimental method in 2009, then put forward that the qualified rate can be up to $98 \%$ as long as the recycling way of waste plastic chip is reasonable and normative, and it could make very high reuse value. The delamination damage of waste plastic chip is a common problem which influences chips' reuse value and reliability, so the research of chip interface delamination has been the core content in the waste plastic chip reusability study.

Aiming at the interface delamination fracture problems of waste plastic chip, the exponential Cohesive Zone Model (CZM) was used to analyze delamination crack of two phase interface in chip interior, and the chip interface delamination was analyzed by adopting finite element simulation method with the CZM as a guide. Finally, the simulation results were verified to be 
effective through the WPCB disassembly experiment, and concrete countermeasures of WPCB disassembly technology to reduce the chip delamination were put forward.

\section{The Cohesive Zone Model}

Traditional interface delamination fracture research was conducted using fracture mechanics simulation method, thus it needed to set the preset crack in simulation model. Due to the position and size of preset crack were set subjectively, it often brought great influence on the accuracy of calculation results ${ }^{[5]}$. In addition, we can also use the cohesion theory to describe the fracture behavior of material. The crack formation and extension phenomenon is analyzed with the tension displacement law of cohesion theory, and it doesn't need preset crack in simulation process, so this is very suitable for the fracture problems of fixed crack propagation path ${ }^{[6]}$. The delamination phenomenon of plastic chip mainly occurs in the interface between different materials, and the crack formation and extension path is relatively stationary. Therefore, the cohesion theory method was adopted to study the delamination fracture problems of plastic chip in this paper.

In the delamination fracture process of plastic chip, a transition zone generates in the interface between two phase materials. This paper used the edge-opened crack as example to study the fracture process, as shown in Fig.1(a). There is a transition zone between the original interface and free surface in the tensile fracture zone, which is called cohesive zone. The mechanical properties of material in cohesive zone are different from other interfaces of plastic chip. For the mechanical properties of cohesive zone, it is described with the tension displacement law. Among numerous cohesion models, the tension displacement law of exponential CZM most conforms to the Van Der Waals force form, and gets high accuracy to solve the problems of composite interface fracture ${ }^{[7,8]}$. So the exponential CZM was adopted to study the interface delamination fracture problems of plastic chip in this paper.

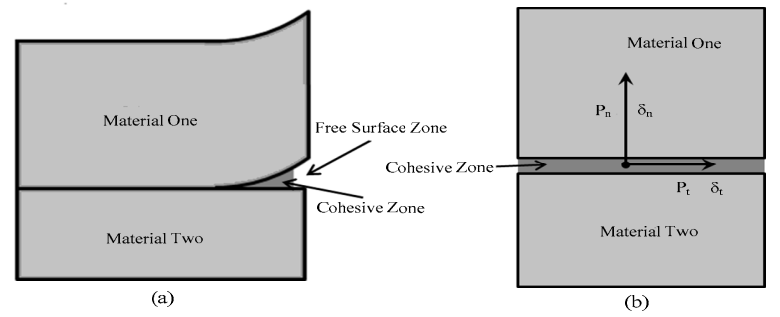

Fig.1 Cohesive zone of plastic chip delamination interface

The cohesive zone of delamination crack in plastic chip is shown in Fig.1(b), making an analysis of one point in the cohesive zone, the received tension of the point can be decomposed to a normal tension $P_{n}$ which is perpendicular to the interface and a tangential tension $P_{t}$ which is parallel to the interface. The interface's fracture displacement $\delta$ can be also decomposed to a normal displacement $\delta_{n}$ and a tangential displacement $\delta_{t}$. The relation between the fracture energy $\varphi(\delta)$ of exponential CZM and the interface's fracture displacement $\delta$ is shown in Eq. $1^{[8]}$.

$$
\varphi(\delta)=e \sigma_{\max } \delta_{t \max }\left[1-\left(1+\frac{\delta_{n}}{\delta_{t \max }}\right) e^{\frac{-\delta_{n}}{\delta_{t \max }}} e^{\frac{-\delta_{t}^{2}}{2 \delta_{n \max }^{2}}}\right]
$$

In formula (1), $e$ is the base of natural logarithms, $\sigma_{\max }$ is the biggest value for the normal tension $P_{n}$ of the cohesive zone, $\delta_{n \max }$ is the corresponding normal displacement under the action of the maximum normal tension $\sigma_{\max }, \delta_{t \max }$ is the corresponding tangential displacement under the action of the maximum tangential tension $\tau_{\max }$.

After getting the partial derivative of fracture energy $\varphi(\delta)$ to normal displacement $\delta_{n}$, the relation between the normal tension $P_{n}$ and the normal displacement $\delta_{n}$ is shown in Eq.2.

$p_{n}\left(\delta_{n}\right)=\frac{e \sigma_{\max } \delta_{n}}{\delta_{t \max }} e^{\frac{-\delta_{n}}{\delta_{t \max }}} e^{\frac{-\delta_{t}^{2}}{2 \delta_{n \max }^{2}}}$

Similarly, after getting the partial derivative of fracture energy $\varphi(\delta)$ to tangential displacement $\delta_{t}$, 
the relation between the tangential tension $P_{t}$ and the tangential displacement $\delta_{t}$ is shown in Eq.3.

$$
p_{t}\left(\delta_{t}\right)=\frac{e \sigma_{\max } \delta_{t}}{\delta_{n \text { max }}^{2}}\left(\delta_{t \text { max }}+\delta_{n}\right) e^{\frac{-\delta_{n}}{\delta_{t \max }}} e^{\frac{-\delta_{t}^{2}}{2 \delta_{n \max }^{2}}}
$$

In the delamination fracture process of plastic chip, it is assumed that tangential displacement $\delta_{t} \rightarrow 0$, normal displacement $\delta_{n} \rightarrow \infty$. According to the Eq.1, the fracture energy $\Psi_{n}$ for getting interface fracture of plastic chip in normal direction is shown in Eq.4.

$\psi_{n}=e \sigma_{\max } \delta_{t \max }$

Similarly, it is assumed that normal displacement $\delta_{n} \rightarrow 0$, tangential displacement $\delta_{n} \rightarrow \infty$. The fracture energy $\Psi_{t}$ for getting interface fracture of plastic chip in tangential direction is shown in Eq.5.

$\psi_{t}=e \sigma_{\max } \delta_{t \max }$

According to the Eq.4 and Eq.5, occurring interface fracture of plastic chip in normal direction and tangential direction requires the same fracture energy.

It is assumed that normal displacement $\delta_{n}=0$, tangential displacement $\delta_{t}=\delta_{\text {tmax }}$. According to the Eq.3, the maximum tangential tension $\tau_{\max }$ is shown in Eq.6.

$\tau_{\max }=\sqrt{e} \sigma_{\max } \frac{\delta_{t \max }}{\delta_{n \max }}$

Research shows that the main force causing interface delamination of plastic chips is interfacial shear ${ }^{[9]}$, so this paper focused on the use of tangential tension-displacement $\left(P_{t}-\delta_{t}\right)$ formula to simulate the delamination phenomenon of plastic chips. According to the Eq.3, making normal displacement $\delta_{n}=0$, the relation between the normalization tangential tension $\Pi_{p}=P_{t} / \tau_{\max }$ and the normalization tangential displacement $\Delta_{\tau}=\delta_{t} / \sqrt{2} \delta_{n \max }$ is shown in Eq.7.

$\Pi_{p}\left(\Delta_{\tau}\right)=\sqrt{2 e} \Delta_{\tau} e^{-\Delta_{\tau}^{2}}$

The curve of Eq.7 is shown in Fig.2. It shows that when $\Delta_{\tau}=\sqrt{2} / 2$, namely $\delta_{t}=\delta_{n \max }$, the tangential tension of cohesive zone reaches the maximum value $\tau_{\max }$, then with the tangential displacement $\Delta_{\tau}$ increasing, interface delamination fracture of plastic chip will occur. When $\Delta_{\tau}=3 \sqrt{2} / 2$, namely $\delta_{t}=3 \delta_{n \max }$, the tangential tension $P_{t}$ of cohesive zone decreases to approximately $5 \%$ of maximum tangential tension $\tau_{\max }$, the interface of plastic chip is almost completely fractured at this time, and it is serious delamination damage to plastic chip.

Above is the exponential cohesion mathematical model. For the interface delamination fracture problems of plastic chips, when material interface is different, the parameters of CZM and the tension-displacement curve is also different. Thus, for a particular delamination interface of plastic chips, which should have the only parameters of CZM, i.e., the fracture energy $\left(\Psi_{n}=\Psi_{t}\right)$ and the displacement $\left(\delta_{n \max }, \delta_{t \max }\right)$ under the action of maximum tension in all directions are uniquely determinate. In the actual analysis of the plastic chips' delamination crack, the parameters of CZM should be calculated beforehand depending on the mechanical properties of interface two-phase material in plastic chip. Then the resulting model parameters are substituted into the CZM, and the next step of delamination simulation is following.

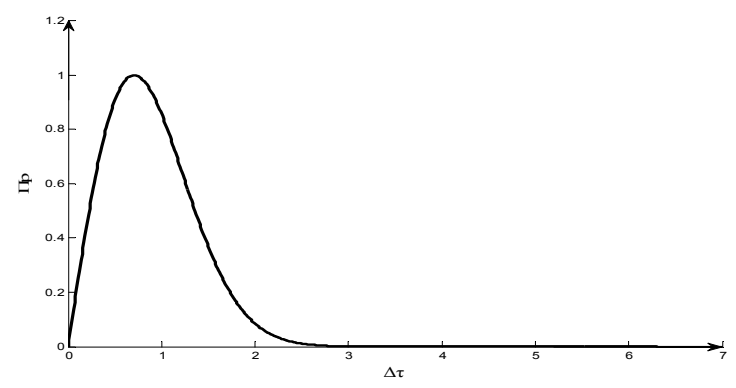

Fig.2 Relation curve of tangential tension $\Pi_{p}$ and tangential displacement $\Delta_{\tau}$ 


\section{Experimental Determinations for Parameters of Cohesive Zone Model}

Prior to the delamination simulation of plastic chip by CZM, the model parameters for corresponding interface of plastic chip should be obtained. A combination of experiments and simulation methods were adopted to determine the model parameters in this paper, which was shown in Fig.3. The fracture displacement parameters of CZM can be obtained through the crack observation experiment. The fracture energy parameters of CZM can be obtained through the method of combining strength test and crack propagation simulation.

The substrate-molded plastic interface of old Quad Flat Pack (QFP) plastic chip was used as the measuring object in this paper, and the following parameters were measured separately.

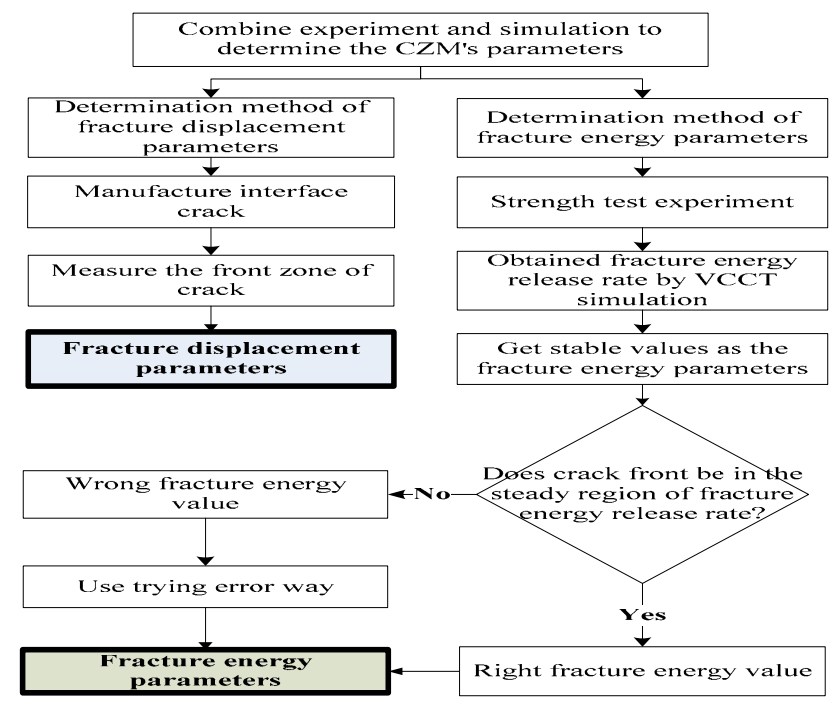

Fig.3 Determination process of Cohesive Zone Model's parameters

The Interface Fracture Displacement Parameters of Plastic Chips

The fracture displacement parameters were measured by the method of manually manufacturing and measuring interface cracks of plastic chips. Due to the width magnitude of the interface crack is $1 \mu \mathrm{m}$, it cannot be measured with the naked eyes, so a scanning electron microscope is necessary to realize the observations and measurement. Strong thermal shock was formed in the interface by applying high temperature load to the old plastic chips, and the minor cracks were produced due to the different thermal expansion coefficients of the two-phase materials at chips' interfaces. Then the processing of cutting, grinding, polishing etc. were done to the chips, and the electron microscopy sections perpendicular to the direction of interface were produced. Finally, a scanning electron microscopy was used to measure the front zone of minor crack to get the fracture displacement. As shown in Fig.4, the maximum normal fracture displacement $\delta_{n \max }$ of substrate - molding plastic interface is $0.0003 \mathrm{~mm}$, so the maximum tangential fracture displacement $\delta_{\text {tmax }}$ is also $0.0003 \mathrm{~mm}$. Thereby the corresponding fracture displacement parameters of CZM for the old QFP plastic chip's substrate-molded plastic interface were obtained.

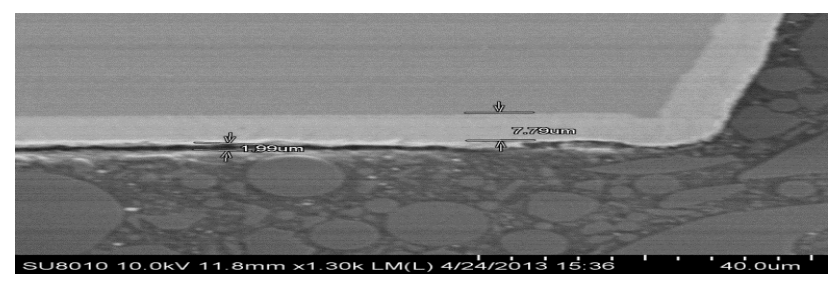

Fig.4 Front zone of substrate-molded plastic interface crack

\section{The Fracture Energy Parameters}

For the interfacial fracture energy parameters of old plastic chips, firstly the shear strength of substrate-molded plastic interface was obtained by strength test experiment, then the shear strength values were loaded as the boundary conditions of finite element simulation, and the interfacial fracture energy parameters for old plastic chips were obtained through the pre-crack simulation method. In order to accurately measure the shear strength of substrate - molding plastic interface, 
the excess parts of the chip were processed by small feed rate intermittent grinding, and the junction surface between the molded plastic and substrate was exposed. In the experiment, one side of the experimental sample's junction surface was fixed, and junction surface of the other end was cut by cutting head, as shown in Fig.5(a). The shear test device was shown in Fig.5(b). The stress-rupture curve of substrate-molded plastic interface was shown in Fig.6, it showed that the fracture behavior of substrate-molded plastic interface occurred instantaneously, and no material yield process happened, which meant to be brittle fracture. In experimental process, several groups of data were measured, and it showed that the results' distribution was dispersive. So the average value of several data groups was taken as the final result, and the average shear strength of substrate-molded plastic interface was approximately $0.98 \mathrm{MPa}$.

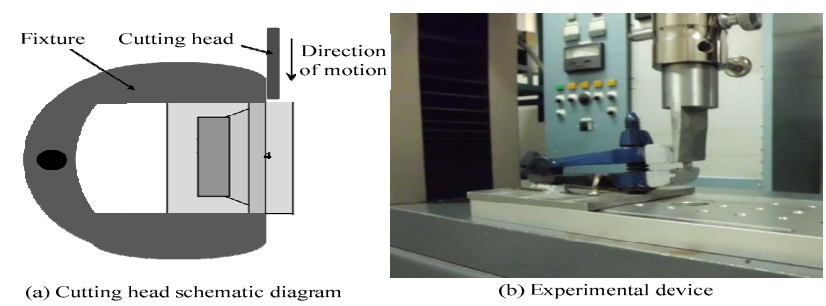

Fig.5 Plastic chip shear test experimental device

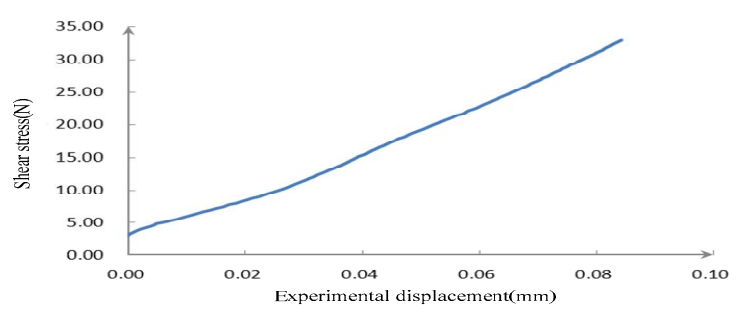

Fig.6 Stress-rupture curve of substrate-molded plastic interface

According to the average shear strength of substrate-molded plastic interface obtained in the experiment, the fracture energy release rate under different preset crack length was calculated by ansys 13.0 based on Virtual Crack Closure Technique (VCCT). Finite element simulation model is shown in Fig.7, the upper material is molded plastic, and the lower material is copper substrate. The boundary conditions and loads of simulation were set accordance with the shear experiment, the simulation parameters of various materials were as shown below, elastic modulus of molded plastic $E_{1}=16 \mathrm{GPa}$, Poisson's ratio $\gamma_{1}=0.3$, elastic modulus of copper substrate $E_{2}=120 \mathrm{GPa}$, Poisson's ratio $\gamma_{2}=0.34$. In the simulation, under the effect of maximum shear stress, the relation curve between preset crack length and fracture energy release rate for crack expansion is shown in Fig.8. When preset crack length is greater than $0.5 \mathrm{~mm}$, the fracture energy release rate for crack extension tends to a stable value, and the cohesive zone of preset crack has occurred fracture. At this point the crack front is in steady region of energy release rate, so the stable value of fracture energy release rate is the old chip's substrate-molded plastic interface fracture energy $\Psi_{n}=\Psi_{t}=0.94 \mathrm{~J} / \mathrm{m} 2$.

Thereby all the necessary parameters of CZM were obtained, after substituting the above parameters into the model, the next step was delamination simulation of plastic chip.

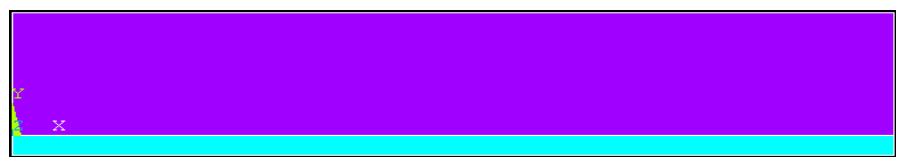

Fig.7 Finite Element Simulation Model 


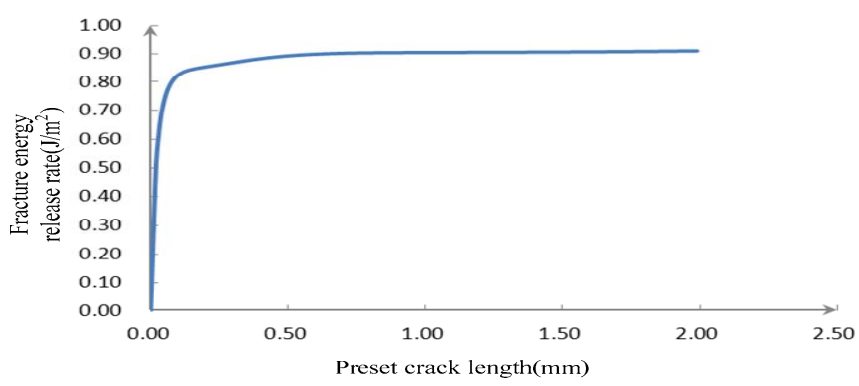

Fig.8 Relation curve of preset crack length and the fracture energy release rate

\section{Delamination Simulations of Plastic Chips}

The main factors affecting the interface delamination of old plastic chips included chip's internal structure, material's physical property parameters, temperature and humidity in chip's external environment, and interface strength of chip. Therefore, in order to minimize the error between the analog value and the true value, this paper considered all the factors in the simulation. Due to the structure of plastic chip is symmetrical, the simulation model just needs to create a half of two-dimensional structure. In order to improve the efficiency of simulation, the model omits the pin portion of chip edge. Each component material's physical property parameters of simulation model are shown in Table1.

Table1 Simulation parameters of materials

\begin{tabular}{ccccc}
\hline Material & Modulus of elasticity $(\mathrm{MPa})$ & Poisson's ratio & Thermal expansion coefficient $\left(10^{-6}\right)$ & Moisture strain $\varepsilon_{H}$ \\
\hline Molded plastic & 16000 & 0.3 & 76 & 0.00702 \\
Copper substrate & 120000 & 0.34 & 16.9 & 0 \\
Die & 169500 & 0.278 & 3.4 & 0 \\
Die attach & 8960 & 0.25 & 41 & 0 \\
\hline
\end{tabular}

After establishing the simulation structural model, based on the corresponding CZM parameters of old QFP plastic chip's substrate-molded plastic interface previously acquired above, added the CZM units to all the interfaces of two-phase material in the chip model. The added CZM parameters included normal (tangential) fracture energy $0.94 \mathrm{~J} / \mathrm{m}^{2}$, normal fracture displacement $0.0003 \mathrm{~mm}$, and tangential fracture displacement $0.0003 \mathrm{~mm}$. The resulting two-dimensional mesh model is shown in Fig.9.

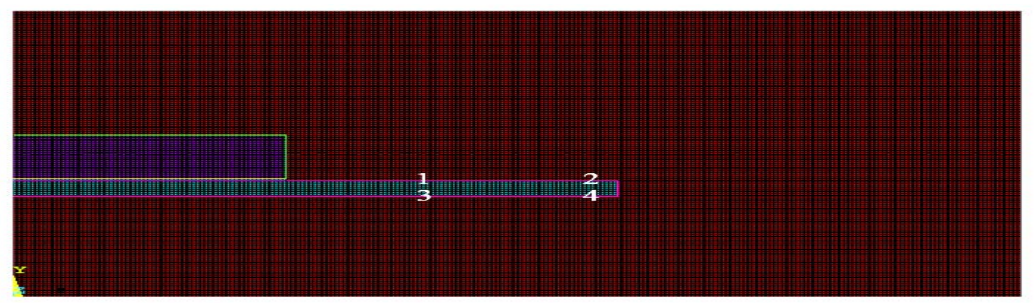

Fig.9 Finite element mesh model of plastic chip

Aim at the main factors affecting the interface delamination of plastic chips, this paper designed comparative simulation groups under different temperatures $\left(\mathrm{A}: 200^{\circ} \mathrm{C}, \mathrm{B}: 260^{\circ} \mathrm{C}\right.$ ) and different humidity (C: indoor humidity, D: $85^{\circ} \mathrm{C} 85 \% \mathrm{RH}$ environment moisture absorption $168 \mathrm{~h}$ ) conditions. In the simulation process, the fracture displacements of four locations (Zone1 4) marked in the old plastic chip's substrate-molded plastic interface (as shown in Figure9) were mainly observed. The fracture simulation is shown in Fig.10, it is for experimental group B-C which is similar to the rest of experimental groups, so this paper no longer enumerates others. The obtained simulation results are shown in Table2, comparing the simulation results in each group, when the chips were in the conditions of high temperature $260{ }^{\circ} \mathrm{C}$ or $85{ }^{\circ} \mathrm{C} 85 \% \mathrm{RH}$ environmental conditions moisture 
absorption 168h, the chips' interfaces completely occurred delamination phenomenon. The results indicated that the heating temperature and ambient humidity of old plastic chips seriously affected their internal interfaces delamination, so it's necessary to reduce the heating temperature as much as possible in the process of waste PCB disassembly for components reuse. Meanwhile, prior to the PCB disassembly, to reduce the delamination rate of plastic chips in waste PCB disassembly experiment as much as possible, the boards should be roasted and be processed by air drying to remove the absorbing moisture in plastic chips' interior.

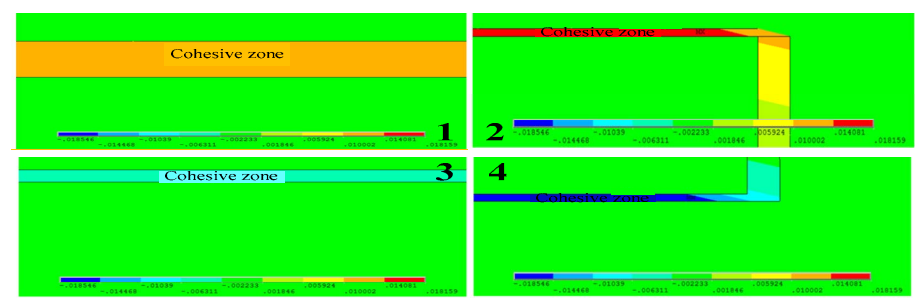

Fig.10 Fracture simulation results of four locations (group B-C)

Table2 Simulation results of comparing experiment groups

\begin{tabular}{|c|c|c|c|c|c|c|c|c|}
\hline \multirow{2}{*}{$\begin{array}{l}\text { Location } \\
\text { number }\end{array}$} & \multicolumn{2}{|c|}{ Group A-C } & \multicolumn{2}{|c|}{ Group B-C } & \multicolumn{2}{|c|}{ Group A-D } & \multicolumn{2}{|c|}{ Group B-D } \\
\hline & $\begin{array}{c}\text { Fracture } \\
\text { displacement }\end{array}$ & Delamination & $\begin{array}{c}\text { Fracture } \\
\text { displacement }\end{array}$ & Delamination & $\begin{array}{c}\text { Fracture } \\
\text { displacement }\end{array}$ & Delamination & $\begin{array}{c}\text { Fracture } \\
\text { displacement }\end{array}$ & Delamination \\
\hline 1 & 0.479 & - & 100 & + & 170 & + & 148 & + \\
\hline 2 & 2.85 & - & 140 & + & 240 & + & 351 & + \\
\hline 3 & 1.31 & - & 63 & + & 170 & + & 155 & + \\
\hline 4 & 2.97 & - & 180 & + & 320 & + & 460 & + \\
\hline
\end{tabular}

\section{Disassembly Experiments of Waste Printed Circuit Boards}

According to the results analysis of old plastic chip delamination simulation, in order to get better WPCB disassembly technology, we use self-developed WPCB disassembly equipment to dismantle a number of discarded computer motherboards, which were divided into four groups of comparison experiments. The equipment is shown in Fig.11(a).

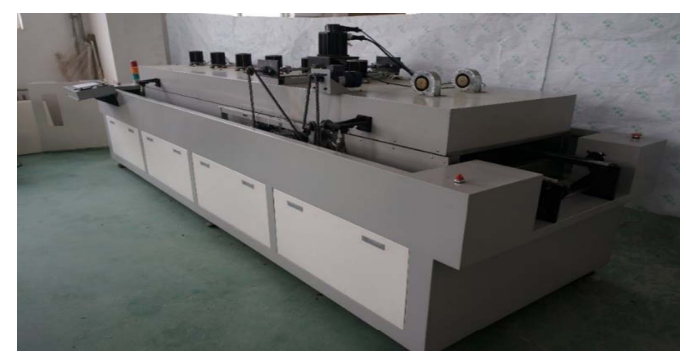

(a) WPCB disassembly equipment

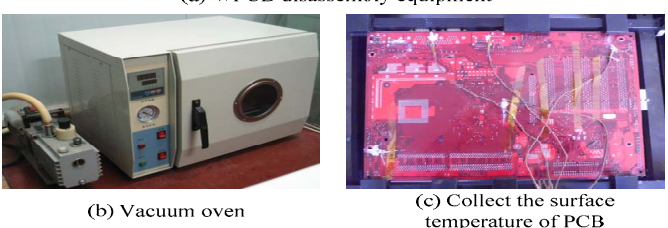

Fig.11 Disassembly experiment equipment

The four groups of WPCB disassembly comparative experiments were as follows: 1) The original group, selected 15 untreated WPCB and used the way of cutting pins to dismantling the old plastic chips on the boards. 2) The low temperature group, selected 15 untreated WPCB and dismantled them at the temperature of $200^{\circ} \mathrm{C}$. 3) The high temperature group, selected 15 untreated WPCB and dismantled them at the temperature of $220^{\circ} \mathrm{C}$. 4) The pre-baking group, selected 15 untreated WPCB and dried them in a $85^{\circ} \mathrm{C}$ vacuum oven (as shown in Fig.11(b)) for 48h, then took them out and dismantled them immediately at the temperature of $200^{\circ} \mathrm{C}$. Due to the disassembly equipment's 
preset temperature was different from the PCB's surface temperature during the experiment, to real-time monitor the changes of PCB's surface temperature, an eight-channel temperature collection module was used to track and collect the temperature of eight points at upside and downside board ,as shown in Fig.11(c). The PCB's surface temperature in dismantling process was shown in Fig.12.

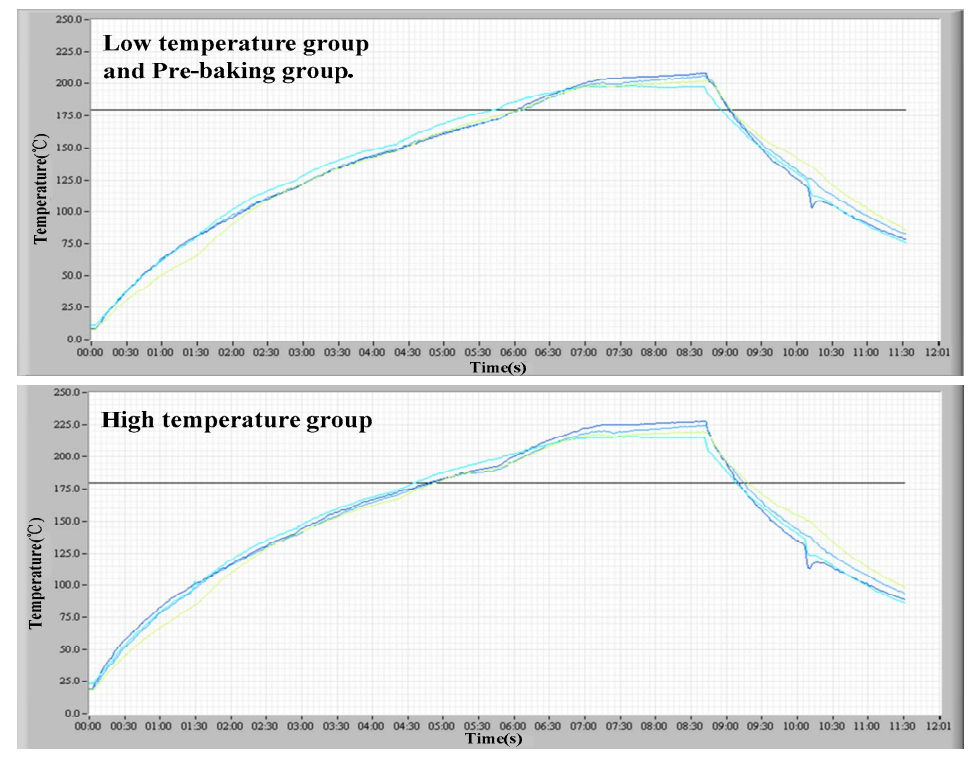

Fig.12 PCB surface temperature monitoring curves in dismantling process

Marked the waste plastic chips dismantled from above four groups of WPCB disassembly experiments, and used the ultrasound $\mathrm{C}$-scan imaging method for detecting delamination morphology of chips' interfaces to determine whether delamination occurred. The ultrasound C-scan images of chips in the experiments were shown in Figure13, the Fig.13(a) was corresponding to plastic chips that interface delamination didn't occur, and Fig.13(b) was corresponding to plastic chips that interface delamination occurred.

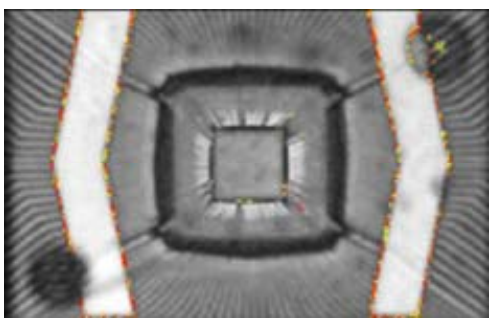

(a) No delamination

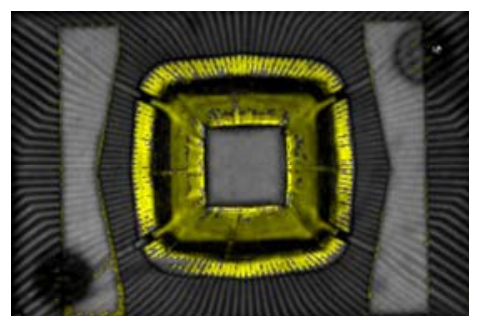

(b) Appearing delamination

Fig.13 Ultrasound C-scan images of old QFP chips

Chip delamination statistics results for the four groups of WPCB disassembly experiments were shown in Table3. From the statistical results, the delamination rate of plastic chips in original group was low to $7.8 \%$, which represented the delamination rate of old plastic chips before dismantling. The delamination rate of plastic chips in low temperature group was $10.6 \%$, which indicated that the delamination rate increased by $2.8 \%$ comparing to the delamination rate before dismantling at the temperature of $200^{\circ} \mathrm{C}$. The delamination rate of plastic chips in high temperature group was $14 \%$, which increased by $3.4 \%$ than the low temperature group. The delamination rate of plastic chips in pre-baking group was $10.4 \%$, which slightly decreased by $0.2 \%$ than the low temperature group. The results showed that, for the WPCB long-term storage in a dry room, due to the amount of moisture absorption was small, pre-baking process did not play a significant role in the impact of delamination rate. Thus in actual industrial operations, in order to save the cost of dismantling, we should depend on the environment around WPCB to determine whether it is necessary to add the pre-baking process. The dismantling temperature had a great impact on the delamination of old plastic chips, so in the premise of ensuring the dismantling rate, dismantling temperature should be 
lowered to reduce the delamination of plastic chips, like this can achieve WPCB's high value recycling for components reuse.

Table3 Delamination statistics results of chips

\begin{tabular}{cccc}
\hline Experiment Group & Chip Type & Quantity & Average Delamination Rate \\
\hline Original group & QFP、PLCC、SOP & 51 & $7.8 \%$ \\
Low temperature group & QFP、PLCC、SOP & 47 & $10.6 \%$ \\
High temperature group & QFP、PLCC、SOP & 50 & $14 \%$ \\
Pre-baking group & QFP、PLCC、SOP & 48 & $10.4 \%$ \\
\hline
\end{tabular}

\section{Conclusions}

This paper studied the delamination problems of chips in the waste plastic chips reuse technology research, built the CZM of delamination crack, and obtained the parameters of CZM by method of combining experiment and simulation. The CZM was used for simulation analysis of waste plastic chips, then the delamination influencing rule of the temperature and humidity factors for the plastic chips was summarized. Finally the correctness of delamination simulation results was verified through the WPCB disassembly experiments, then discussed and put forward optimized WPCB disassembly technology for component reuse.

1) Aim at the problem that calculation accuracy of traditional interface crack simulation methods for two-phase material is insufficient, the exponential cohesion theory which best met the Van Der Waals force form was used to analyze the interface crack, and the accuracy of analysis results were improved.

2) In the CZM's analysis, the crack surface measuring experiment and fracture mechanics finite element simulation method were used to get the parameters of old plastic chips' substrate-molded plastic interface, the problem of difficultly confirming CZM's parameters was solved.

3) The heating temperature and humidity of old plastic chip had a very large impact on the delamination of internal interface, the higher temperature, the greater amount of moisture absorption, the more prone to occur delamination in the dismantling process.

4) In WPCB's industrial dismantling process, to minimize energy consumption and dismantling costs, we should depend on the environmental temperature and humidity of WPCB to determine whether it is necessary to add the pre-baking process for PCB.

\section{Acknowledgements}

The project supported by the National High Technology Research \& Development Program (863 Program) of China, project number: 2013AA040207, 2013 AA040205.

\section{References}

[1] Liu Fuzhong, Tian Hui, Xiang Dong, et al. Circular Economy in Household Electrical Appliance Industry [G]. Beijing: China Light Industry Press, 2010.

[2] Ding Xiaoyu, Liu Xueping, Xiang Dong, et al. Research on delamination defect of plastic IC packages in waste components reuse processes [J]. Proceedings of the fourth world conference on maintenance. 2008

[3] Guo Xiuying, Xiang Dong, Duan Guanghong, et al. Investigation into Reusability of Disassembled Chip[J]. Microelectronics. 2009(5):714-717

[4] Guo Xiuying, Xiang Dong, Duan Guanghong, et al. A Method of WEEE Recycle and Reuse Oriented for Material Recycle and Parts Reuse [J]. Environmental Science and Technology. 2009,32(8):115-119. 
[5] Fan Xuejun, Suhir E. Moisture Sensitivity of Plastic Packages of IC Devices [J]. Boston, MA: Springer Science+Business Media, LLC, 2010.

[6] Huang Liugang. The Analysis of Cohesive Zone Model and User-defined Subroutine Development in Finite Element Method [D]. Zhengzhou: Zhengzhou University, 2010.

[7] Zhang Jun. The Application of Interface Stress and Cohesion Model in Interface Mechanics [M]. Zhengzhou: Zhengzhou University Press, 2011.

[8] P. X X, Needleman. Void nucleation by inclusion debonding in a crystal matrix [J]: Modelling and Simulation in Materials Science and Engineering, 1993: 1, 111 132.

[9] Long Danfeng. Research on the Delamination Mechanism and the Reuse-Oriented Disassembling Method of Plastic-Encapsulated Chip on EOL PCB [D]. Beijing: Tsinghua University, 2013. 